\author{
Seong Mi Moon \\ Yun Soo Hong \\ Dae Sang Lee \\ Chi Ryang Chung
}

\section{Purpura fulminans on the nose with septic abortion}

Received: 17 December 2014

Accepted: 24 December 2014

Published online: 24 January 2015

(C) Springer-Verlag Berlin Heidelberg and ESICM 2015

\section{S. M. Moon · Y. S. Hong}

Department of Internal Medicine, Samsung Medical Center, Sungkyunkwan University School of Medicine, Seoul, South Korea

\section{S. Lee - C. R. Chung}

Department of Critical Care Medicine, Samsung Medical Center, Sungkyunkwan University School of Medicine, Seoul, South Korea

A 39-year-old female presented with shock with a purple nose. She had been admitted in another hospital for management of an incompetent internal orifice of the cervix and had undergone a McDonald operation at 19 weeks of gestational age. Two weeks later, she had a fever, and ultrasonographic evaluation revealed intrauterine fetal death. The dead fetus was delivered with oxytocin, and the patient immediately went into shock. At that time, there was an abrupt skin color change to purple only on the nose. Under the impression of septic abortion, broad-spectrum antibiotics were administered. Laboratory findings showed disseminated intravascular coagulation (DIC). Renal dysfunction ensued so continuous renal replacement therapy was started. Two days later, Escherichia coli was identified from the blood and vaginal discharge cultures. After 7 days of intensive care, she had completely recovered from the septic shock. The purple nose was diagnosed as purpura fulminans (PF) and treated with prostaglandin E1. Then it returned to normal.

$\mathrm{PF}$ is defined as rapidly progressing cutaneous hemorrhage and necrosis of the skin, which is a clinical sign of DIC, and probable vascular wall infection, which is usually associated with sepsis. PF is most commonly associated with meningococcal sepsis, varicella and less commonly pneumococcal infections. PF with nasal involvement is known to be rare.

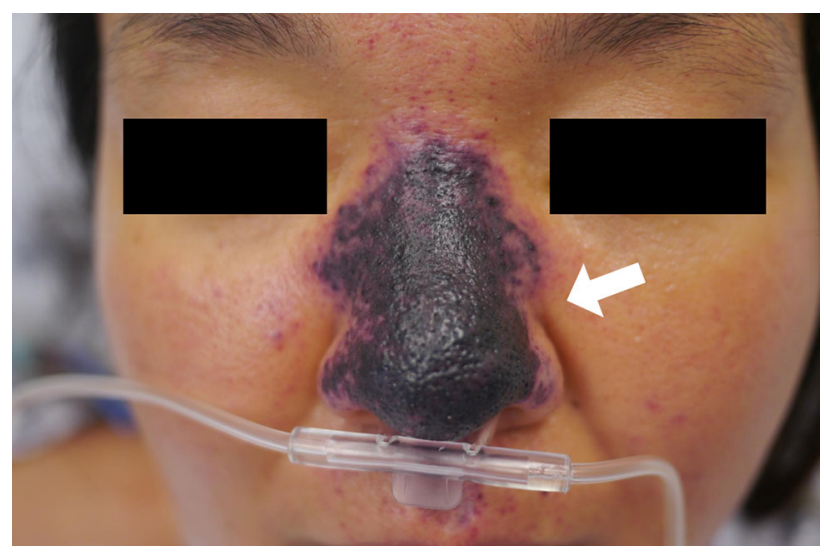

